OPEN ACCESS

Edited by:

Robert L. Lins, Independent Researcher,

Antwerp, Belgium

Reviewed by:

Etienne Lengline,

Assistance Publique Hopitaux

De Paris, France

Tomás José González-López, Burgos University Hospital, Spain

*Correspondence:

Bing Han

hanbing_li@sina.com

${ }^{+}$These authors have contributed equally to this work

Specialty section: This article was submitted to Pharmaceutical Medicine and

Outcomes Research,

a section of the journal

Frontiers in Pharmacology

Received: 13 July 2020 Accepted: 15 October 2020

Published: 16 November 2020

Citation:

Zuo W, Zhang B, Ruan J, Chen M and Han B (2020) Correlation of the Plasma

Concentration of Eltrombopag With

Efficacy in the Treatment of Refractory Aplastic Anemia: A Single-Centre

Study in China.

Front. Pharmacol. 11:582625. doi: 10.3389/fphar.2020.582625

\section{Correlation of the Plasma} Concentration of Eltrombopag With Efficacy in the Treatment of Refractory Aplastic Anemia: A Single-Centre Study in China

\author{
Wei Zuo ${ }^{1 \dagger}$, Bo Zhang ${ }^{1 \dagger}$, Jing Ruan ${ }^{2}$, Miao Chen ${ }^{2}$ and Bing Han $^{2 *}$ \\ ${ }^{1}$ Department of Pharmacy, Peking Union Medical Colleague Hospital, Chinese Academy of Medical Science, Beijing, China, \\ ${ }^{2}$ Department of Hematology, Peking Union Medical Colleague Hospital, Chinese Academy of Medical Science, Beijing, China
}

Background and purpose: Eltrombopag (ELT) can be effective in the treatment of relapse/refractory aplastic anemia (AA) patients. Responses and adverse drug reactions (ADRs) differed greatly among individuals treated at the same dosage of ELT.

Methods: Patients diagnosed with nonsevere aplastic anemia (NSAA) between January 2018 and January 2019 in Peking Union Medical Colleague Hospital who were refractory to immunosuppressive therapy were treated with ELT and followed up for at least 6 months. Plasma concentrations of ELT were detected by high-performance liquid chromatography-mass spectrometry after at least two months of ELT treatment and treatment at the same dosage for at least 2 weeks. The dose-concentration, concentration-response and concentration-ADR relationships were evaluated.

Results: Among the 72 patients treated with ELT during the study period, 44 patients with complete data were enrolled. Six (13.6\%) were males, and 38 were females (86.4\%), with a median age of 54 years [interquartile range (IQR): 38.5-63]. At the time the ELT plasma concentration was detected, the median dosage of ELT was 75 (IQR 50-100) mg/d, the median time of total ELT exposure was 3 (IQR 2.0-6.0) months, and 37 (70.5\%) patients had responded to ELT. The median concentration of ELT was $10.4 \mu \mathrm{g} / \mathrm{ml}$ (IQR 3.7-24.4 $\mu \mathrm{g} / \mathrm{ml})$. The concentration of ELT was positively correlated with the daily dose of ELT $(r=0.68, p<0.001)$. Multivariate logistic regression analysis showed that the risk of inefficacy of ELT at a concentration between 11.2 and $15.2 \mu \mathrm{g} / \mathrm{ml}$ was $0.028-$ fold $(95 \% \mathrm{Cl}$ : $0.001-0.864 ; p=0.041$ ) of that at a concentration between 3.2 and $7.2 \mu \mathrm{g} / \mathrm{ml}$. The cutoff value for the concentration of ELT showing efficacy was $12.50 \mu \mathrm{g} / \mathrm{ml}$ according to the receiver operation characteristic curve. A higher risk of ADR was related to a longer total exposure to ELT $(p=0.012)$. Although the correlation was not significant, the odds ratio increased with the ELT concentration, suggesting that it was possible that an elevated risk of ADR was correlated with the ELT blood concentration.

Conclusion: ELT is effective for the treatment of NSAA and has acceptable side effects. The plasma concentration of ELT was correlated with the dose and the effects of ELT.

Keywords: eltrombopag, non-severe aplastic anemia, refractory, plasma concentration, efficacy 


\section{INTRODUCTION}

Few therapeutic options are available for patients with aplastic anemia (AA) who are ineligible for transplantation and refractory to immunosuppressive therapy. Eltrombopag (ELT), a small molecule thrombopoietin mimetic, has been proven to induce trilinear hematopoietic responses in relapsed/refractory AA patients (Desmond et al., 2014). The results of a phase II trial examining ELT monotherapy in refractory severe aplastic anemia patients showed a $40 \%$ response rate after 3-4 months of therapy (Olnes et al., 2012; Desmond et al., 2014), which led to the approval of ELT as a monotherapy for relapsed/refractory SAA in the United StatesA and Europe (Novartis, 2017; Novartis, 2018). However, the use of ELT is only approved for idiopathic thrombocytopenic purpura (ITP) in China by the National Medical Products Administration; for AA, the use of ELT is still "off-label" (Zhu et al., 2019). Therefore, it is not covered by national health insurance for the indication of AA. Currently, the use of ELT in Chinese patients with AA is still limited; moreover, the high price of ELT for long-term medication might further restrict its application.

Many studies have focused on the relationship between the dosage of ELT and its efficacy (Gibiansky et al., 2011; Matthys et al., 2011). However, the clinical responses and adverse drug reactions (ADRs) differed greatly among individuals, even those treated with the same dosage (Choy et al., 2016). To date, there have been few studies demonstrating the clinical significance of the blood concentration of ELT in patients with AA in daily clinical practice. Therapeutic drug monitoring is a branch of clinical pharmacology that specializes in the measurement of drug concentrations in blood, aiming at improving patient care by individually adjusting the dose of drugs (Oellerich et al., 2017). Early identification of populations for which a drug may be inappropriate might be helpful in adjusting the treatment plan and reducing the burden and expense. Given that the patients' responses the to the drug differed dramatically, we would like to clarify whether plasma concentrations of ELT could be an indicator of its efficacy and/or adverse events.

\section{MATERIALS AND METHODS}

\section{Study Population}

This is a prospective study. From January 2018 to January 2019, outpatients in Peking Union Medical Colleague Hospital (PUMCH, Beijing, China) diagnosed with nonsevere aplastic anemia (NSAA) and refractory to immunosuppressive therapy (cyclosporine A for at least 6 months) were treated with ELT. Cyclosporine A (CsA) had been stopped for at least 6 months before ELT treatment. No other drugs, such as tacrolimus, danazol, or stanozolol, were used during the experimental period. Blood samples from patients who had been treated with ELT for at least 2 months and had been treated at the same dosage for at least 2 weeks were included in the final analysis. The enrolled patients received at least four visits (once a month) to evaluate the efficacy and side effects. All participants and/or their legal guardians signed written informed consent forms before blood was drawn for tests. This study was approved by the Ethical Committee of $\mathrm{PUMCH}$ and was conducted in accordance with the Declaration of Helsinki.

\section{Clinical Data Collection}

ELT was started at $25 \mathrm{mg}$ /day for 2 weeks, and the dosage was increased every 2 weeks to a maximum of $100 \mathrm{mg} /$ day and then tapered gradually (by $25 \mathrm{mg} /$ day every 3 months) after the best response was achieved. Baseline information, including the clinical and laboratory data, was recorded. Symptoms (including side effects) and signs were recorded every month after treatment by one of the researchers, who was blinded to the outcome of the analysis. Laboratory assessments (complete blood count with differential and serum chemistry profiles, ECG or other tests if needed) were performed in our hospital and very occasionally in other local hospitals.

Patients were followed up for at least 6 months after ELT treatment, and the outcome was determined either from the medical documents or by telephone interviews of the patients or their relatives.

\section{Blood Sample Collection and Testing of the Plasma Eltrombopag Concentration}

All patients enrolled had been exposed to ELT for at least 2 months and had been stable at the current dosage for 2 weeks to ensure a steady blood concentration. On the day of the blood draw, patients were asked to undergo ELT treatment under the supervision of the researchers to minimize the errors caused by time variation in the drawing of blood. Two hours later, blood samples were collected via the forearm vein into tubes containing K3-EDTA and were immediately placed in ice water. The plasma samples were separated and then stored at $-80^{\circ} \mathrm{C}$ in polypropylene tubes until analysis. Plasma from healthy volunteers was obtained as a normal control.

The plasma concentrations of ELT were assayed by highperformance liquid chromatography-tandem mass spectrometry (Maddela et al., 2014). This assay can detect the entire range of ELT concentrations from 100 to $50,000 \mathrm{ng} / \mathrm{ml}$. The imprecision and inaccuracy were less than 20 and $18 \%$, respectively, whereas the intra-assay error was less than $10 \%$. The ELT reference standard (99.69\% pure) was obtained from MedChemExpress (United States, HY15306A). ELT 13C4 (99.66\% pure) was employed as an internal standard and was obtained from TLC Pharmaceutical Standards Ltd.

\section{Criteria for Evaluation}

Complete remission (CR) and partial remission (PR) were defined according to previous literature (Marsh et al., 2009). Briefly, 1) CR was defined as normal hemoglobin (HGB), neutrophils $\left(\right.$ ANC) $>1.5 \times 10^{9} / \mathrm{L}$, and platelets $(\mathrm{PLT})>100 \times$ $10^{9} / \mathrm{L}$; and 2) PR was defined as A) stoppage of blood transfusion (in patients who were previously blood transfusion-dependent); $\mathrm{B})$ restoration of at least one lineage to normal or 2-fold increased compared with the baseline; and C) HGB $>30 \mathrm{~g} / \mathrm{L}(<60 \mathrm{~g} / \mathrm{L}$ before treatment), ANC $>0.5 \times 10^{9} / \mathrm{L}$ (less than $0.5 \times 10^{9} / \mathrm{L}$ before 
treatment), PLT $>20 \times 10^{9} / \mathrm{L}$ (less than $20 \times 10^{9} / \mathrm{L}$ before treatment) compared with the baseline. No response (NR) was defined as not having any of the above responses.

The causality assessment of ADRs was performed with a standardized algorithm by two doctors based on the Common Terminology Criteria for Adverse Events Version 5.0 (U.S. Department of Health and Human Services, 2017).

\section{Statistical Analysis}

Data are expressed as the median with the interquartile range (IQR) for continuous variables and as percentages/frequency (\%) for categorical variables. Normally distributed data were compared by Student's t-test, while non-normally distributed data were compared by the Mann-Whitney U test. Categorical data were analyzed by the chi-square test. Logistic regression analysis was conducted to determine the relationship between ELT efficacy or adverse drug reactions (ADRs) and ELT concentrations by adjusting for potential confounding factors. $p$-values and 95\% CIs were two-sided. $p<0.05$ was considered statistically significant. Data were analyzed using SPSS version 17.0 .

\section{RESULTS}

\section{Patient Demographics}

Very few patients with SAA were treated with ELT. To reduce the heterogeneity of the patient population, we focused only on patients with NSAA. From January 2018 to January 2019, 72 patients with NSAA who were refractory to CsA received ELT treatment. Among them, 10 patients were exposed to ELT for less than 2 months and were switched to other medications for various reasons. Eight patients refused to give blood for ELT concentration determination, and 10 patients were lost to followup within 6 months of ELT treatment. Overall, 44 patients were included in the final analysis, with a median age of 54 years (IQR: $38.8-63.0)$. Of the 44 patients, 6 (13.6\%) were males, while 38 were females ( $86.4 \%)$. To avoid selection bias, we double-checked the data of the included and excluded patients. There were no significant clinical, hematological or treatment differences between the screened and included patients. Before the initiation of the ELT regimen, patients had been treated with CsA for a median of 12 months (6-36), and the rate of transfusion was $2(0-6)$ and $2(0-7)$ units/month for red blood cells and 2 (0-4) and $2(0-4)$ for platelets at the time of diagnosis and ELT treatment initiation, respectively. The median hemoglobin count was $69.0 \mathrm{~g} / \mathrm{L}$ (IQR 56.3-91.0 g/L), with a median platelet count of $9.5 \times 10^{9} / \mathrm{L}\left(\right.$ IQR $\left.6.8-14.0 \times 10^{9} / \mathrm{L}\right)$, a median absolute neutrophil count of $1.16 \times 10^{9} / \mathrm{L}$ (IQR $0.8-1.6$ ), and a median reticulocyte count of $51.5 \times 10^{9} / \mathrm{L}(\mathrm{IQR} 47.1-71.6)$ at the time of ELT treatment initiation. At the time blood was obtained for concentration determination, the median dosage of ELT was 75 (IQR 50.0-100.0) $\mathrm{mg} / \mathrm{d}$, and the median time of total ELT exposure was 3 (IQR 2.0-4.0) months. Accordingly, the median hemoglobin count at the same time was 80.5 (IQR 65.0-105.0) $\mathrm{g} / \mathrm{L}$, with a median platelet count of 26.0 (IQR $14.0-19.0) \times 10^{9} / \mathrm{L}$ and a median absolute neutrophil count of 1.6 (IQR 1.6-1.7) $\times$
$10^{9} /$ L. All the baseline clinical characteristics are shown in Table 1.

\section{Eltrombopag Treatment and Response}

Patients were followed up for a median of 8 (IQR: 7.0-10.0) months, and the longest follow-up period was 12 months after ELT treatment. According to their response at the time of blood concentration determination, patients were divided into the response group (those who had achieved at least PR) and the nonresponse group (patients with NR). There was no difference in the baseline characteristics in the groups with or without response (Table 1). Moreover, the total time of exposure to ELT and the time at the current dosage were the same between the two groups $(p=0.37$ and $p=0.54$, respectively).

One month after ELT, an overall response was observed in $61.4 \%(27 / 44)$ of patients. The response rate increased over time. By the end of the follow-up period, one patient with NR had achieved PR, and two patients with PR had achieved CR, whereas one patient with PR relapsed when ELT was still being used, resulting in final odds ratio (OR), CR and PR rates of $70.5 \%$ (31/44), 27.3\% (12/44), and 43.2\% (19/44), respectively. The hematologic cell lineage response at different time points is shown in Figure 1. Three patients stopped ELT treatment due to a lack of effectiveness or relapse at 8, 12, and 12 months. No death or clone evolution was noticed during the follow-up period.

\section{Clinical Significance of the Plasma Concentration of Eltrombopag}

All enrolled patients were tested to determine the plasma concentration $2 \mathrm{~h}$ after receiving ELT (peak concentration). The median concentration of ELT was $10.4 \mu \mathrm{g} / \mathrm{ml}$ (IQR $3.7-24.4 \mu \mathrm{g} / \mathrm{ml})$. Potential clinical characteristics that may have influenced the concentration of ELT were summarized and calculated in the multivariate regression analysis (Table 2). The regression equation showing the relationship between the ELT dose and concentration was successfully determined ( $Y=$ $0.7482+0.1554 X, p<0.00)$, and the blood concentration of ELT was positively correlated with the daily dose of ELT $(r=0.68, p<$ 0.001 , Figure 2 ). We also found a positive relationship between the ELT plasma concentration and the magnitude of platelet improvement $(p<0.05)$ and lymphocyte improvement $(p<$ 0.05 ), as shown in Figure 2. Since no patients had severe impairment of kidney or liver function after ELT, no relevant significant change in the plasma concentration was noticed in our study, as indicated in Table 1. No correlation between the plasma concentration of ELT and other clinical characteristics was found (Table 2).

\section{The Correlation Between the Plasma Concentration and the Efficacy of Eltrombopag}

Due to the small number of patients, individual variables with a $p$-value less than 0.20 in Table 1 were included in the following multivariate regression analysis. After identifying other 
TABLE 1 | Clinical characteristics of the patients enrolled.

\begin{tabular}{|c|c|c|c|c|}
\hline & Total & Response group & Non-response group & $p$ \\
\hline Number of patients (n, \%) & $44(100.0)$ & $31(70.5)$ & $13(29.5)$ & - \\
\hline \multicolumn{5}{|l|}{ Demographic characteristics } \\
\hline Gender, n. (\%) ${ }^{\mathrm{a}}$ & & & & 0.83 \\
\hline Male & $6.0(13.6)$ & $4.0(12.9)$ & $2.0(15.4)$ & - \\
\hline Female & $380.0(86.4)$ & $27.0(87.1)$ & $11.0(84.6)$ & - \\
\hline Age, years, $[\mathrm{QQR}]^{\mathrm{b}}$ & $54.0[38.5,63.0]$ & $54.0[38.5,62.0]$ & $53.0[39,64.0]$ & 0.680 \\
\hline Weigh, kg $[\mathrm{IQR}]^{\mathrm{b}}$ & $63.8[56.5,67.0]$ & $65.0[57.5,68.5]$ & $60.0[55.3,65.5]$ & 0.25 \\
\hline Height, cm $[\mathrm{IQR}]^{\mathrm{b}}$ & $163.0[160.0,165.0]$ & $162.5[160.0,165.0]$ & $163.0[160.0,165.0]$ & 0.63 \\
\hline Previous CsA median months (range) & $12[6,36]$ & $11[6,32]$ & $14[7,36]$ & 0.73 \\
\hline \multicolumn{5}{|l|}{ Red blood cells (U/month) } \\
\hline Time on diagnosis & $2[0-6]$ & $2[0-4]$ & $2[0-6]$ & 0.91 \\
\hline Time on ELT onset & $2[0-7]$ & $2[0-6]$ & $2[0-7]$ & 0.81 \\
\hline \multicolumn{5}{|l|}{ Platelet (U/month) } \\
\hline Time on diagnosis & $2(0-4)$ & $2[0-3]$ & $2[0-4]$ & 0.83 \\
\hline Time on ELT onset & $2(0-4)$ & $2[0-4]$ & $2[0-4]$ & 0.96 \\
\hline \multicolumn{5}{|l|}{ Biological characteristics } \\
\hline \multicolumn{5}{|l|}{ Before ELT } \\
\hline Hemoglobin, g/L [IQR] ${ }^{\mathrm{C}}$ & $69.0[56.3,91.0]$ & $70.0[50.5,89.0]$ & $65.0[57.0,91.0]$ & 0.57 \\
\hline Neutrophils, absolute count, $\times 10^{9} / \mathrm{L}[\mathrm{IQR}]^{\mathrm{C}}$ & $1.2[0.8,1.6]$ & $1.2[0.8,1.7]$ & $1.1[1.0,1.4]$ & 0.69 \\
\hline Platelet count, $\times 10^{9} / \mathrm{L}[\mathrm{IQR}]^{\mathrm{C}}$ & $9.5[6.8,14.0]$ & $9.0[7.0,13.0]$ & $10.0[6.0,19.0]$ & 0.78 \\
\hline Reticulocyte counts, $\times 10^{9} / \mathrm{L}[\mathrm{IQR}]^{\mathrm{c}}$ & $51.5[47.1 .71 .6]$ & $52.0[46.5,70.5]$ & $51.5[50.2,71.6]$ & 0.88 \\
\hline $\mathrm{PNH}$ Clone $(\mathrm{n}, \%)^{\mathrm{a}}$ & & & & 0.95 \\
\hline No PNH clone & $41.0(93.2)$ & $29.0(93.5)$ & $12.0(92.3)$ & - \\
\hline With small PNH clone & $3.0(6.8)$ & $2.0(6.5)$ & $1.0(7.7)$ & - \\
\hline $\mathrm{SGPT}, \mathrm{U} / \mathrm{L},[\mathrm{IQR}]^{\mathrm{C}}$ & $47.0[22.0 .92 .0]$ & $39.0[16.5 .88 .5]$ & $52.0[32.0,110.0]$ & 0.45 \\
\hline TBIL, $\mu \mathrm{mol} / \mathrm{L},[\mathrm{IQR}]^{\mathrm{C}}$ & $7.2[3.9,11.1]$ & $5.9[2.8 .7 .8]$ & $7.1[7.2 .10 .8]$ & 0.39 \\
\hline Scr, $\mu \mathrm{mol} / \mathrm{L},[\mathrm{IQR}]^{\mathrm{C}}$ & $59.5[26.0-152.0]$ & $45.0[26.0 .97 .0]$ & $69.0[34.0,105.0]$ & 0.31 \\
\hline $\mathrm{LDH}, \mathrm{U} / \mathrm{L},[\mathrm{IQR}]^{\mathrm{C}}$ & $262.0[209.0,328.0]$ & $304.0[271.5,354.0]$ & $257.0[207.5,262.0]$ & 0.59 \\
\hline $\mathrm{SF}, \mathrm{ng} / \mathrm{ml},[\mathrm{IQR}]^{\mathrm{C}}$ & $573.0[53.8,1423.5]$ & $637.0[35.0,3783.0]$ & $502.0[65.0,2782.0]$ & 0.41 \\
\hline \multicolumn{5}{|l|}{ At the time of blood taken for concentration } \\
\hline Hemoglobin, g/L [IQR] $]^{c}$ & $80.5[65.0,105.0]$ & $83.0[70.0,107.5]$ & $79.0[61.0,101.0]$ & 0.32 \\
\hline Neutrophils, absolute count, $\times 109 / \mathrm{L}[\mathrm{IQR}]^{\mathrm{C}}$ & $1.6[1.6 .1 .7]$ & 1.8 [1.3.2.2] & $1.1[1.1 .1 .7]$ & 0.04 \\
\hline Platelet count, $\times 109 / \mathrm{L}[\mathrm{IQR}]^{\mathrm{C}}$ & $26.0[14.0 .49 .0]$ & $40.5[17.0 .84 .5]$ & $14.0[9.0 .26 .0]$ & 0.00 \\
\hline Reticulocyte counts, $\times 10^{9} / \mathrm{L}[\mathrm{IQR}]^{\mathrm{c}}$ & $51.5[50.1 .70 .3]$ & $51.0[49.5,71.5]$ & $51.5[50.0,70.1]$ & 0.86 \\
\hline White blood cell, absolute count, $\times 109 / L[I Q R]^{c}$ & $3.4[2.9-4.2]$ & $3.4[2.9 .3 .9]$ & $3.6[3.0 .4 .3]$ & 0.71 \\
\hline Mean corpuscular volume, fL $[\mathrm{IQR}]^{\mathrm{C}}$ & $105.2[98.4,111.1]$ & $105.9[97.9,112.3]$ & $102.0[100.0,107.3]$ & 0.24 \\
\hline Lymphocytes, absolute count, ×109/L [IQR] ${ }^{c}$ & $1.4[2.0 .1 .0]$ & $1.2[1.1 .1 .8]$ & $2.1[0.9 .2 .8]$ & 0.15 \\
\hline \multicolumn{5}{|l|}{ Transaminases, $\mathrm{n}(\%)^{\mathrm{a}}$} \\
\hline Reduced & $3.0(6.8)$ & $3.0(9.7)$ & $0(0)$ & 0.68 \\
\hline Normal & $24.0(54.5)$ & $16.0(51.6)$ & $8.0(61.5)$ & - \\
\hline Elevated & $17.0(38.6)$ & $12.0(38.7)$ & $5.0(38.5)$ & - \\
\hline \multicolumn{5}{|l|}{ Serum creatinine, n. (\%)a } \\
\hline Normal & $34.0(77.3)$ & $23.0(74.2)$ & $11.0(84.6)$ & 0.37 \\
\hline Elevated & $10.0(22.7)$ & $8.0(25.8)$ & $2.0(15.4)$ & - \\
\hline \multicolumn{5}{|l|}{ ELT at the time of blood concentration } \\
\hline Months from ELT start $[\mathrm{IQR}]^{\mathrm{c}}$ & $3.0[2.0 .4 .0]$ & $3.0[2.0 .4 .0]$ & $2.3[2.0 .4 .0]$ & 0.97 \\
\hline Weeks with the current dose $[\mathrm{IQR}]^{\mathrm{C}}$ & $3.0[2.0 .4 .0]$ & $3[2.0 .4 .0]$ & $3[2.0 .4 .0]$ & 1.00 \\
\hline Patients with different dose, $\mathrm{n}(\%)^{\mathrm{a}}$ & $75.0[50.0 .75 .0]$ & $75.0[50.0 .75 .0]$ & $75.0[50.0 .75 .0]$ & 1.00 \\
\hline $25 \mathrm{mg} / \mathrm{d}$ & $3.0(6.8)$ & $2.0(6.4)$ & $1.0(7.7)$ & - \\
\hline $50 \mathrm{mg} / \mathrm{d}$ & $14.0(31.8)$ & $10.0(32.3)$ & $4.0(30.8)$ & - \\
\hline $75 \mathrm{mg} / \mathrm{d}$ & $17.0(38.6)$ & $12.0(38.7)$ & $5.0(38.5)$ & - \\
\hline $100 \mathrm{mg} / \mathrm{d}$ & $10.0(22.7)$ & $7.0(22.6)$ & $3.0(23.1)$ & - \\
\hline \multicolumn{5}{|l|}{ Plasma concentration, $\mu \mathrm{g} / \mathrm{ml}$} \\
\hline Min, Max & $3.7,24.4$ & $3.7,24.4$ & $5.2,20.5$ & - \\
\hline Median $\left[\mathrm{IQR}^{\mathrm{C}}\right.$ & $10.4[8.2 .13 .8]$ & $12.0[8.8 .14 .6]$ & $9.5[8.1-10.4]$ & 0.13 \\
\hline Graded concentrations, $\mathrm{n}(\%)^{\mathrm{a}}$ & & & & 0.08 \\
\hline$[3.2-7.2] \mu \mathrm{g} / \mathrm{ml}$ & $8.0(18.2)$ & $5.0(16.1)$ & $3.0(23.1)$ & - \\
\hline$[7.2-11.2] \mu \mathrm{g} / \mathrm{ml}$ & $15.0(34.1)$ & $8.0(25.8)$ & $7.0(53.8)$ & - \\
\hline$[11.2-15.2] \mu \mathrm{g} / \mathrm{ml}$ & $13.0(29.5)$ & $11.0(35.5)$ & $2.0(15.4)$ & - \\
\hline$[>15.2] \mu \mathrm{g} / \mathrm{ml}$ & $8.0(18.2)$ & $7.0(22.6)$ & $1.0(7.7)$ & - \\
\hline ELT follow-up time, month, $[I Q R]^{c}$ & $8.0[7.0 .10 .0]$ & $9.0[7.0 .10 .0]$ & $8.0[6.0,11.5]$ & 0.80 \\
\hline
\end{tabular}

IQR, interquartile range; $A A$, aplastic anemia; CSA, cyclosporine; CR, Complete remission; PR, partially remission; OR, overall remission; SGPT, serum glutamic-pyruvic transaminase; TBIL, total bilirubin; Scr, serum creatinine; $L D H$, lactate dehydrogenase; SF, serum ferritin. Comparisons of variable between the different concentration groups.

${ }^{a}$ Pearson's chi-square test.

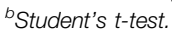

${ }^{c}$ Nonparametric Mann-Whitney test. 

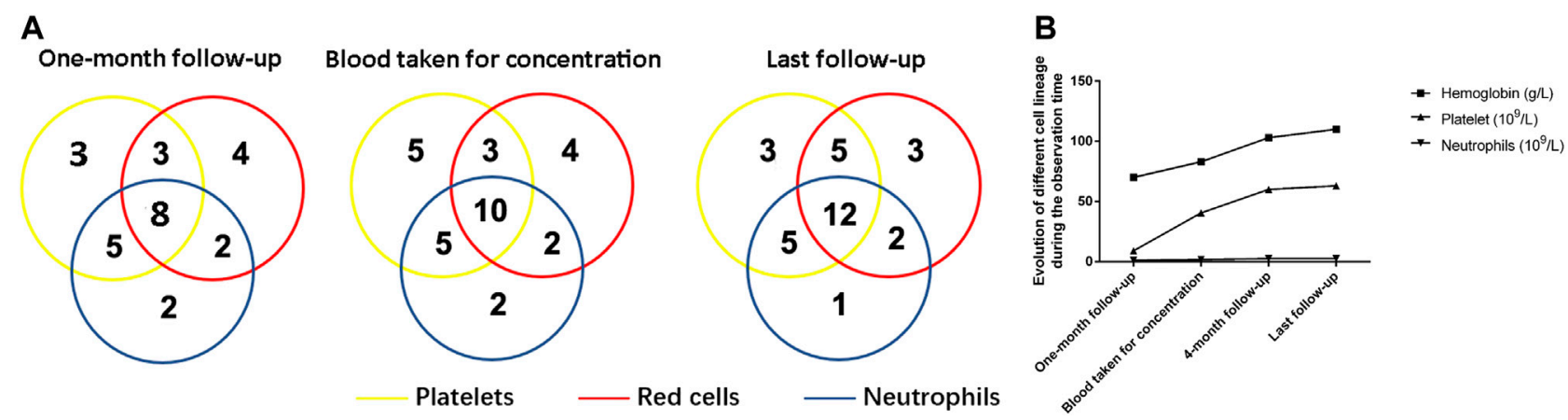

FIGURE 1 | Hematologic improvements caused by Eltrombopag at different time points. (A) The Venn diagrams show the number of patients with unilineage, bilineage, and trilineage hematologic responses at the time when the blood concentration was determined and at the time of last follow-up. (B) A cumulative line chart showing hemoglobin/platelet/neutrophil evolution during the observation time.

influencing factors with a multivariate logistic regression analysis, a significant correlation between ELT efficacy and concentration (Table 3) was found. The OR decreased as the concentration of ELT increased, suggesting an elevated response rate at a higher ELT plasma concentration. A concentration between 11.2 $15.2 \mu \mathrm{g} / \mathrm{ml}$ was associated with a 0.03 -fold (95\% CI, $0.00-0.86$; $p=0.04$ ) lower risk of ineffectiveness compared with the reference concentration. However, when the concentration of ELT was above $15.2 \mu \mathrm{g} / \mathrm{ml}$, the response rate was slightly decreased compared to that associated with concentrations between 11.2 and $15.2 \mu \mathrm{g} / \mathrm{ml}$. To determine the cut-off values of the relationship of the ELT concentration to efficacy, ROC curves were generated with AUCs of 0.65 (95\% CI, 0.48-0.83) (Figure 3). The diagnostic specificity of ELT inefficacy increased, and the sensitivity decreased with increasing ELT concentration. Youden's index identified the optimal cut-off point as $12.5 \mu \mathrm{g} / \mathrm{ml}$.

TABLE 2 | Correlations of clinical characteristics and plasma concentrations of ELT.

\begin{tabular}{lcc}
\hline Number & Pearson's $\boldsymbol{r}$ & $\boldsymbol{p}$ \\
\hline Demographic characteristics & & \\
Age, years & 0.08 & 0.23 \\
Weigh, $\mathrm{kg}$ & 0.11 & 0.67 \\
Height, $\mathrm{cm}$ & 0.05 & 0.38 \\
$\quad$ Gender & -0.11 & 0.24 \\
Biological characteristics & & \\
$\quad$ Before ELT & & \\
$\quad$ Hemoglobin, g/L & -0.42 & 0.67 \\
$\quad$ Neutrophils, absolute count, $\times 10^{9} / \mathrm{L}$ & -0.10 & 0.68 \\
$\quad$ Platelet count, $\times 10^{9} / \mathrm{L}$ & -0.24 & 0.20 \\
Transaminases & -0.00 & 0.88 \\
$\quad$ Serum creatinine & 0.25 & 0.86 \\
Magnitude of hematological improvement (at the time of TDM) & & \\
$\quad$ Neutrophils, absolute count, $\times 10^{9} / L$ & 0.11 & 0.41 \\
Platelet count, $\times 10^{9} / L$ & 0.58 & 0.04 \\
$\quad$ Lymphocytes, absolute count, $\times 10^{9}$ & 0.51 & 0.05 \\
ELT treatment & & \\
$\quad$ Months from ELT started & 0.16 & 0.36 \\
Months with the current dose & 0.27 & 0.08 \\
Dosage, mg/day & 0.68 & 0.00
\end{tabular}

At this threshold, the sensitivity was $51.6 \%$, and the specificity was $84.6 \%$.

\section{Correlation Between the Plasma Concentration of Eltrombopag and Adverse Drug Reactions}

Adverse effects were reported in $17 / 44$ (38.6\%) patients. The two most common ADRs reported were lower limb oedema (11.4\%, $5 / 44)$ and the elevation of transaminase $(6.8 \%, 3 / 44)$. Other reported ADRs included skin rash $(4.5 \%, 2 / 44)$, creatine elevation $(4.5 \%, 2 / 44)$, nausea/vomiting $(2.3 \%, 1 / 44)$, skin segmentation $(2.3 \%, 1 / 44)$, tachycardia $(2.3 \%, 1 / 44)$, oropharyngeal blister $(2.3 \%, 1 / 44)$ and musculoskeletal pain $(2.3 \%, 1 / 44)$. All ADRs were grade one. Multivariate logistic regression analysis was performed to determine the possible risk factors for ADRs, including gender, age, ELT concentration, renal/liver function and other biological characteristics. From the results, we can see that a higher risk of ADRs was related to a longer total exposure time to ELT ( $p=$

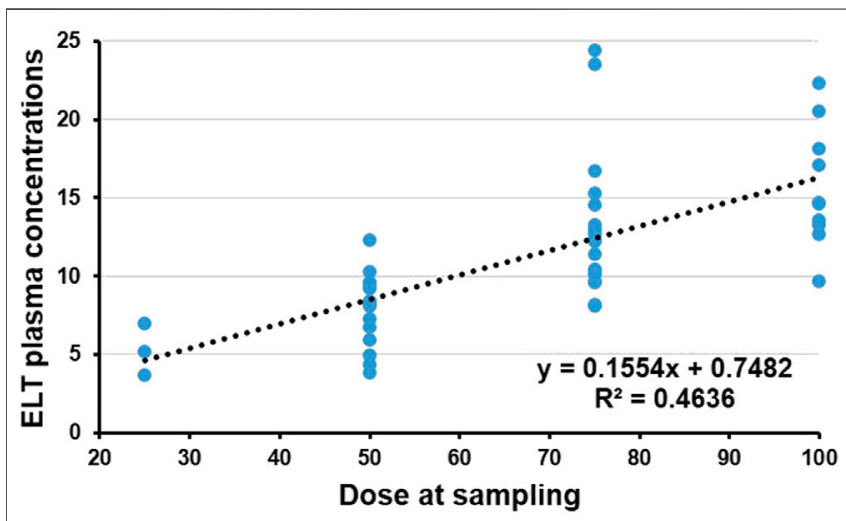

FIGURE 2 | Plasma concentration and dose of Eltrombopag (ELT). The regression equation of the relationship between the dose of ELT and concentration was successfully determined $(Y=0.7482+0.1554 X, p<$ 0.001). The plasma concentration of ELT was significantly positively correlated with the daily dose of ELT $\left(r^{2}=0.4636, p<0.001\right)$. 
TABLE 3 | Multivariate logistic regression analysis of variables associated with inefficacy.

\begin{tabular}{lcc} 
Covariate & \multicolumn{2}{c}{ Ineffective } \\
\cline { 2 - 3 } & OR (CI) & $p$-value \\
\hline Graded concentration, $\mu \mathrm{g} / \mathrm{ml}$ & Ref & - \\
{$[3.2-7.2]$} & $0.33[0.02,7.10]$ & 0.48 \\
{$[7.2-11.2]$} & $0.03[0.00,0.86]$ & $0.04^{\star}$ \\
{$[11.2-15.2]$} & $0.09[0.00,3.18]$ & 0.19 \\
{$[>15.2]$} & & \\
Biological characteristics (at the time of TDM) & $1.15[0.91,1.44]$ & 0.24 \\
Neutrophils, absolute count, $\times 10^{9} / \mathrm{L}$ & $0.87[0.76,0.99]$ & $0.04^{\star}$ \\
Platelet count, $\times 10^{9} / \mathrm{L}$ & $7.85[0.93,66.49]$ & 0.06 \\
Lymphocytes, absolute count, $\times 10^{9}$ &
\end{tabular}

OR: odd ratio; Ref, reference category for the odds ratio. ${ }^{a} \mathrm{p}$-value calculated using multivariate regression analysis. ${ }^{*} \mathrm{p}<0.05$.

0.01). Although no significant relationship was noticed between ADRs and the ELT concentration $(p>0.05)$, the OR increased with the ELT concentration, suggesting a possible elevation of the risk of ADRs with the blood concentration (Table 4). No significant relationships were noticed for other risk factors.

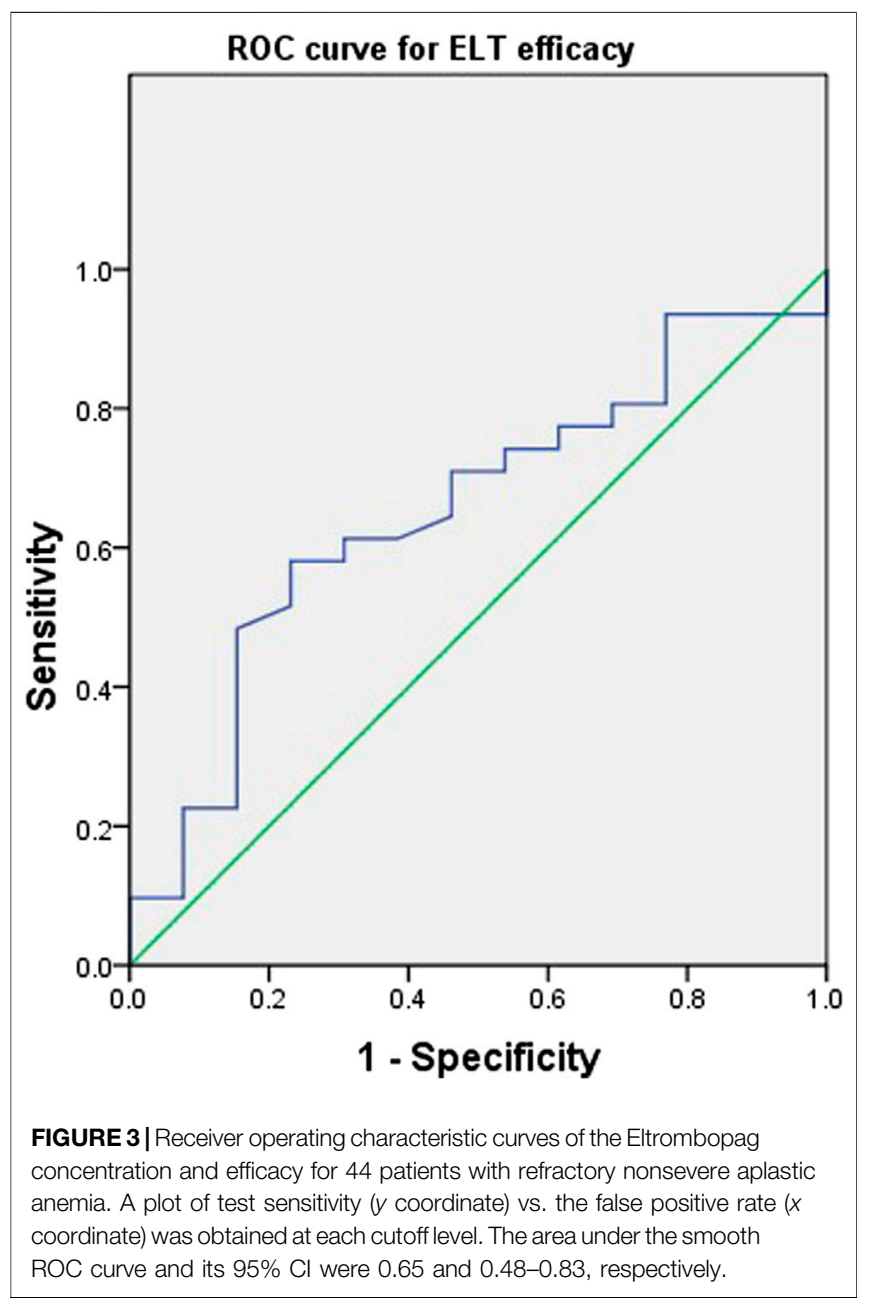

\section{DISCUSSION}

In this study, we selected patients with refractory NSAA who had been treated with ELT for at least 2 months to evaluate the correlation between the plasma concentration and the efficacy of ELT. Those who received ELT for less than 2 months or who withdrew within 2 months of ELT treatment initiation for different reasons (economic insufficiency, NR, side effects, lost follow-up, etc.) were excluded. Some patients refused to sign the consent form for blood testing, especially those with a poor response, which may partly explain the relatively high response rate $(70.5 \%)$ in our cohort compared to that in the literature (Olnes et al., 2012; Desmond et al., 2014; Lengline et al., 2018; Ecsedi et al., 2019). Although the response rate was calculated at the time when blood was obtained to determine the ELT concentration, which may have been too short for an efficacy evaluation, most of the patients had already been exposed to ELT for more than 3 months in our cohort. It has been shown in the literature that most refractory SAA patients responded to ELT within 3 months of treatment initiation (Olnes et al., 2012). Moreover, the follow-up data of such patients showed that only a few showed an increase or decrease in efficacy subsequently, making the time of the blood concentration test a good time for determining the efficacy of ELT. Similar to reports from other investigators, nearly half of the patients had a trilineage response rather than only a platelet response (Desmond et al., 2014).

Although other studies have verified the effectiveness of ELT for refractory AA (Lengline et al., 2018), the optimal dosage and treatment duration have not been well defined so far. To the best of our knowledge, no data are available for determining the concentration-effect relationship of ELT. In most cases, increasing the dosage is an easy solution for patients with a poor response, but this may lead to a high economic burden or additional side effects. All enrolled patients had been exposed to ELT for at least 2 months and were stable at the current dosage for 2 weeks to ensure a steady blood concentration; furthermore, patients were tested to determine the peak concentration $(2 \mathrm{~h}$ after ELT) to minimize the errors caused by variations in the time of the blood draw. Our results showed that the blood

TABLE 4 | Multivariate logistic regression analysis of variables associated with adverse drug reaction.

\begin{tabular}{lcc} 
Covariate & \multicolumn{2}{c}{ Adverse effect } \\
\cline { 2 - 3 } & OR (CL) & p-value ${ }^{\text {a }}$ \\
\hline Gender & $1.861[0.155,22.344]$ & 0.24 \\
Age & $1.023[0.696,1.080]$ & 0.41 \\
Months from ELT started & $1.307[1.060,1.611]$ & $0.01^{*}$ \\
Transaminases & $0.167[0.102,98.362]$ & 0.79 \\
Serum creatinine & $1.192[0.162,7.725]$ & 0.86 \\
Graded concentration, $\mu \mathrm{g} / \mathrm{ml}$ & & \\
$\quad$ [3.2-7.2] & 1 & 0.64 \\
[7.2-11.2] & $7.07[0.22,226.13]$ & 0.27 \\
{$[11.2-15.2]$} & $23.77[0.58,971.45]$ & 0.09 \\
$\quad>15.2]$ & $5.37[0.13,218.35]$ & 0.37
\end{tabular}

OR, odd ratio; Ref, reference category for the odds ratio. ${ }^{a} \mathrm{p}$-value calculated using multivariate regression analysis. ${ }^{*} p<0.05$. 
concentration of ELT was positively correlated with the daily dose of ELT within a certain range of concentrations. Under these circumstances, increasing the dose can increase the concentration of ELT and thus improve the efficacy. However, it seemed that the response rate was slightly decreased in patients with ELT concentrations above $15.2 \mu \mathrm{g} / \mathrm{ml}$ compared with that in patients with concentrations between 11.2 and $15.2 \mu \mathrm{g} / \mathrm{ml}$, illustrating the minimal benefit of substantially increasing the dosage. In addition, we calculated a cut-off point of $12.50 \mu \mathrm{g} / \mathrm{ml}$ for optimal sensitivity and specificity. Although they need to be verified in further studies, these results implied that there is a dose-effect correlation for ELT in the treatment of refractory NSAA.

On the other hand, it has been reported previously that Asians may show slower metabolism of ELT compared with other ethnic groups (Wu et al., 2015), which may suggest that a lower dosage of ELT be used in Asian patients. However, a recent report from Hong Kong showed that higher doses (up to $300 \mathrm{mg}$ ) were tolerable and effective for Chinese patients, although some significant skin pigmentation was observed in patients treated with high doses (Gill et al., 2017; Hwang et al., 2018). However, a few other reports from Japan showed that a median dose of $84 \mathrm{mg}$ (25-100 mg) can achieve an efficacy of $48 \%-55 \%$ (Konishi et al., 2019). In our study, a response could be observed at relatively low doses of $50-100 \mathrm{mg} / \mathrm{day}$ and at a median of 3 months. None of the patients received a dosage greater than $100 \mathrm{mg} /$ day. However, the OR rate was $70.5 \%$, and most of the patients reached the peak concentration that may indicate a favourable response, as shown in our study. We did not monitor the ELT concentration dynamically because the patients were unwilling to undergo frequent blood draws. Only four patients had their ELT concentration rechecked three times during their follow-up period. Three of them had low concentrations the first time blood was obtained and had a poor response at the same time. Neither an increase in their ELT concentration nor an improvement in their response was noticed after a longer period of ELT exposure (checked at 3, 6, and 8 months, respectively) at the same dosage. However, one patient had a high concentration $(75 \mathrm{mg} / \mathrm{d}, 14.2 \mu \mathrm{g} / \mathrm{ml})$ the first time blood was obtained and had a good response to ELT. He maintained a good response after dosage reduction to a final dosage of $50 \mathrm{mg} /$ $\mathrm{d}$, and the concentrations at 6 and 8 months were $11.2 \mu \mathrm{g} / \mathrm{ml}$ and $11.8 \mu \mathrm{g} / \mathrm{ml}$, respectively. Those patients with a lower concentration and a poor response the first time blood was obtained for concentration determination did not show an increase in their ELT concentration or an improvement in their response after exposure to ELT for a longer time when the dose of ELT remained the same, suggesting that the 2 months duration of our experiment may be long enough to predict the effectiveness of ELT at that dosage. Collectively, our data indicated that, similar to CsA concentration monitoring in the treatment of AA (Liang et al., 2011; Philippe et al., 2015), monitoring of the concentration of ELT at certain points might also be helpful for the prediction of effectiveness.

Some factors, such as residual hematopoiesis, telomere length, age, degree of bone marrow hypocellularity, megakaryocytic morphology (absent megakaryocytes vs. reduced megakaryocytes) and even the polyclonal lymphoid marrow percentage, have been demonstrated as predictors of the response to ELT (Desmond et al., 2014; Hwang et al., 2018; Fattizzo et al., 2019). The baseline characteristics were compatible for patients with or without a response in our cohort, which was probably due to the small number of patients and the high lost to follow-up rate, which made our patient population highly selected. Nevertheless, the plasma concentration of ELT might be a potential reference for determining the response as well, as shown in our results.

Elevated transaminase is the most common dose-dependent toxic effect of ELT (Ecsedi et al., 2019), according to published data. Other side effects mentioned in the literature include skin rash, elevated bilirubin, skin pigmentation, nausea, fatigue, cough, diarrhea, headache, and possible clone evolution (Lum and Grainger, 2016; Scheinberg, 2019). In a recent study in Europe, ADRs were reported in $28 \%(51 / 180)$ of patients (Ecsedi et al., 2019). It has been shown that high-dose or long-term use of ELT may cause a higher rate of ADRs (Yang et al., 2017). In our study, approximately $38.6 \%$ of patients (17 in 44) reported ADRs. In accordance with the literature, a higher risk of ADRs was related to a longer total exposure time to ELT. Although the OR seemed to increase with the ELT concentration, suggesting a possible elevated risk of ADR, most of the ADRs were mild and easily controlled without drug suspension. This was probably because all the patients in our study were treated with a relatively low dose of ELT compared with that used in other reports, suggesting that a low dose range may be safer and more effective for Chinese patients.

Our study has some limitations. This was a single-centre study with a relatively small sample size, which might contribute to the uneven distribution of the male/female ratio in our cohort. The patients were not successive, and there might have been selection bias. The loss to follow-up rate was high, which might cause a higher OR rate since patients with poor responses or severe side effects may have been lost. The lack of dynamic monitoring of the concentration and the response rate might limit the evaluation of long-term efficacy and ADRs. However, our study demonstrated the efficacy of ELT in patients with refractory NSAA with acceptable toxicity.

Our study showed that the blood concentration of ELT within a certain range was correlated with the dose of ELT and probably indicated the OR rate. However, a high drug concentration exceeding a certain value might not further improve the efficacy. Long-term exposure to ELT may lead to a higher risk of ADR. The blood concentration of ELT might be associated with ADRs, but the lower and upper limits of the dose of ELT applied in our study might have protected against ADRs. Further studies with a larger number of patients and longer follow-up times with dynamic monitoring are needed.

\section{DATA AVAILABILITY STATEMENT}

The raw data supporting the conclusions of this article will be made available by the authors, without undue reservation.

\section{ETHICS STATEMENT}

The studies involving human participants were reviewed and approved by Ethical Committee of the Peking Union Medical College and 
Chinese Academy of Medical Sciences. The patients/participants provided their written informed consent to participate in this study.

\section{AUTHOR CONTRIBUTIONS}

Drug concentration test: $\mathrm{WZ}$ and BZ; Clinical data collection: WZ, JR, and MC; Project administration, BZ; Supervision, BH; Writing-original draft: WZ; Writing-review and editing: $\mathrm{BH}$.

\section{REFERENCES}

Choy, K. W., Wijeratne, N., and Doery, J. C. G. (2016). Eltrombopag: liver toxicity, kidney injury or assay interference? Pathology 48 (7), 754-756. doi:10.1016/j. pathol.2016.07.011

Desmond, R., Townsley, D. M., Dumitriu, B., Olnes, M. J., Scheinberg, P., Bevans, M., et al. (2014). Eltrombopag restores trilineage hematopoiesis in refractory severe aplastic anemia that can be sustained on discontinuation of drug. Blood 123 (12), 1818-1825. doi:10.1182/blood-2013-10-534743

Ecsedi, M., Lengline, É., Lengline, É., Knol-Bout, C., Bosman, P., Eikema, D.-J., et al. (2019). Use of eltrombopag in aplastic anemia in Europe. Ann. Hematol. 98 (6), 1341-1350. doi:10.1007/s00277-019-03652-8

Fattizzo, B., Kulasekararaj, A. G., Hill, A., Benson-Quarm, N., Griffin, M., Munir, T., et al. (2019). Clinical and morphological predictors of outcome in older aplastic anemia patients treated with eltrombopag. Haematologica 104 (11), e494-e496. doi:10.3324/haematol.2019.216374

Gibiansky, E., Zhang, J., Williams, D., Wang, Z., and Ouellet, D. (2011). Population pharmacokinetics of eltrombopag in healthy subjects and patients with chronic idiopathic thrombocytopenic purpura. J. Clin. Pharmacol. 51 (6), 842-856. doi:10.1177/0091270010375427

Gill, H., Leung, G. M. K., Lopes, D., and Kwong, Y.-L. (2017). The thrombopoietin mimetics eltrombopag and romiplostim in the treatment of refractory aplastic anaemia. Br. J. Haematol. 176 (6), 991-994. doi:10.1111/bjh.14024

Hwang, Y.-Y., Gill, H., Chan, T. S. Y., Leung, G. M. K., Cheung, C. Y. M., and Kwong, Y.-L. (2018). Eltrombopag in the management of aplastic anaemia: real-world experience in a non-trial setting. Hematology 23 (7), 399-404. doi:10. 1080/10245332.2017.1422306

Konishi, A., Nakaya, A., Fujita, S., Satake, A., Nakanishi, T., Azuma, Y., et al. (2019). Evaluation of eltrombopag in patients with aplastic anemia in realworld experience. Leuk. Res. Rep. 11, 11-13. doi:10.1016/j.lrr.2019.03.002

Tsubokura, E., Drenou, B., Peterlin, P., Tournilhac, O., Abraham, J., Berceanu, A., et al. (2018). Nationwide survey on the use of eltrombopag in patients with severe aplastic anemia: a report on behalf of the French reference center for aplastic anemia. Haematologica 103 (2), 212-220. doi:10.3324/haematol.2017.176339

Liang, L. Y., Zhang, L., Jing, L. P., Zhou, K., Wang, X. D., Li, Y., et al. (2011). Effects of concentration of cyclosporine $\mathrm{A}$ on the early response to immunosuppressive therapy in severe aplastic anemia. Zhonghua Xue Ye Xue Za Zhi 32 (11), 766-771 [in Chinese]. doi:10.3760/cma.j.issn.0253-2727.2011.11.010

Lum, S. H., and Grainger, J. D. (2016). Eltrombopag for the treatment of aplastic anemia: current perspectives. Drug Des. Dev. Ther. 10, 2833-2843. doi:10.2147/ DDDT.S95715

Maddela, R., Gajula, R., Pilli, N. R., Siddiraju, S., Maddela, S., and Makula, A. (2014). Liquid chromatography-tandem mass spectrometric assay for eltrombopag in $50 \mu \mathrm{L}$ of human plasma: a pharmacokinetic study. J. Pharmaceut. Biomed. Anal. 98, 68-73. doi:10.1016/j.jpba.2014.04.028

Marsh, J. C. W., Ball, S. E., Cavenagh, J., Darbyshire, P., Dokal, I., Gordon-Smith, E. C., et al. (2009). Guidelines for the diagnosis and management of aplastic anaemia. Br. J. Haematol. 147 (1), 43-70. doi:10.1111/j.1365-2141.2009.07842.x

Matthys, G., Park, J. W., McGuire, S., Wire, M. B., Bowen, C., Williams, D., et al. (2011). Pharmacokinetics and pharmacodynamics clinical pharmacokinetics, platelet response, and safety of eltrombopag at supratherapeutic doses of up to

\section{FUNDING}

This study was supported by grants from Beijing Natural Science Foundation (7192168), the Chinese Academy of Medical Sciences (CAMS) innovation fund for medical sciences (2016I2M-3-004), the Nonprofit Central Research Institute Fund of Chinese Academy of Medical Sciences (2019XK 320047), and the National Natural Science Foundation of China Grants (81974183).

$200 \mathrm{mg}$ once daily in healthy volunteers. J. Clin. Pharmacol. 51 (3), 301-308. doi:10.1177/0091270010368677

Novartis (2017). Promacta prescribing information. Available at: https://www. accessdata.fda.gov/drugsatfda_docs/label/2017/022291s019lbl.pdf. (Accessed July 10, 2020).

Novartis (2018). Revolade product information. Available at: https://www.ema.europa. eu/en/documents/product-information/revolade-epar-product-information_en.pdf (Accessed July 10, 2020).

Oellerich, M., Kanzow, P., and Walson, P. D. (2017). Therapeutic drug monitoring key to personalized pharmacotherapy. Clin. Biochem. 50 (7-8), 375-379. doi:10. 1016/j.clinbiochem.2017.01.007

Olnes, M. J., Scheinberg, P., Calvo, K. R., Desmond, R., Tang, Y., Dumitriu, B., et al. (2012). Eltrombopag and improved hematopoiesis in refractory aplastic anemia. N. Engl. J. Med. 367 (1), 11-19. doi:10.1056/ nejmoa 1200931

Philippe, M., Hénin, E., Bertrand, Y., Plantaz, D., Goutelle, S., and Bleyzac, N. (2015). Model-based determination of effective blood concentrations of cyclosporine for neutrophil response in the treatment of severe aplastic anemia in children. AAPS $J$ 17 (5), 1157-1167. doi:10.1208/s12248-015-9779-8

Scheinberg, P. (2019). Activity of eltrombopag in severe aplastic anemia. Hematol. Am. Soc. Hematol. Educ. Program. 2018 (1), 450-456. doi:10.1182/asheducation2018.1.450

US department of health and human services (2017). Common terminology criteria for adverse events (CTCAE) version 5.0. Available at: https://ctep.cancer.gov/ protocolDevelopment/electronic_applications/docs/CTCAE_v5_Quick_Reference_ 5x7.pdf. (Accessed June 19, 2020).

Wu, K., Thapar, M., Farrell, C., Hayes, S., Guo, H., Hou, M., et al. (2015). Population pharmacokinetic and pharmacodynamic modeling and effects on platelet counts of different dosages of eltrombopag in Chinese patients with chronic primary immune thrombocytopenia. Clin. Therapeut. 7 (37), 1382-1395. doi:10.1016/j.clinthera.2015.03. 024

Yang, R., Li, J., Jin, J., Huang, M., Yu, Z., Xu, X., et al. (2017). Multicentre, randomised phase III study of the efficacy and safety of eltrombopag in Chinese patients with chronic immune thrombocytopenia. Br. J. Haematol. 176 (1), 101-110. doi:10.1111/bjh.14380

Zhang, X.-F., He, H.-L., Wang, S.-Q., Tang, J.-Y., Han, B., Zhang, D.-H., et al. (2019). Current treatment patterns of aplastic anemia in China: a prospective cohort registry study. Acta. Haematol. 142 (3), 162-170. doi:10.1159/000499065

Conflict of Interest: The authors declare that the research was conducted in the absence of any commercial or financial relationships that could be construed as a potential conflict of interest.

Copyright (C) 2020 Zuo, Zhang, Ruan, Chen and Han. This is an open-access article distributed under the terms of the Creative Commons Attribution License (CC BY). The use, distribution or reproduction in other forums is permitted, provided the original author(s) and the copyright owner(s) are credited and that the original publication in this journal is cited, in accordance with accepted academic practice. No use, distribution or reproduction is permitted which does not comply with these terms. 